\title{
ANÁLISIS DE LA INTERCAMBIABILIDAD DE MEDICAMENTOS BIOLÓGICOS EN COSTA RICA
}

\author{
FREDDY ARIAS MORA \\ Régimen Académico Asociado \\ Facultad de Farmacia (Universidad de Costa Rica) \\ freddyariasmora@gmail.com
}

ANDREA B ADILLA JIMÉNEZ

Colegio de Farmacéuticos de Costa Rica

andrea.badilla@misalud.go.cr

RESUMEN: La intercambiabilidad de medicamentos biológicos es un tema que ha generado mucha discusión en el mundo. Debido a la complejidad de estos productos demostrar biosimilitud no es suficiente para establecer su intercambiabilidad. El presente trabajo aborda los conceptos fundamentales sobre la intercambiabilidad, medicamentos biotecnológicos y biosimilares, muestra el panorama internacional de cómo se ha abordado la intercambiabilidad de los medicamentos biosimilares.

PALABRAS CLAVE: medicamentos biológicos, intercambiabilidad, biosimilares.

ABSTRACT: Interchangeability of biological drugs is an issue that has generated much discussion in the world. Due to the complexity of these products, demonstrating biosimilarity is not enough to establish their interchangeability. This paper addresses the fundamental concepts of interchangeability, biotech drugs and biosimilars, shows how the international arena have addressed the interchangeability of biosimilar medicines.

KEYWORDS: biological medicines, interchangeability, biosimilars.

SUMARIO: I. InTROducción. II. MedicAmentos BiolóGicos. III. LA INTERCAMBIABILIDAD DE MEDiCAMENTOS. III.1 Medicamento biotecnológico frente a un medicamento biosimilar. III.2 Intercambiabilidad de los medicamentos biotecnológicos. III.3 El caso de la Eritropoyetina. IV. REGUlACIÓN DE LA INTERCAMBIABILIDAD DE LOS MEDICAMENTOS BIOTECNOLÓGICOS BIOSIMILARES EN LA UNIÓN EUROPEA Y AMÉRICA. V. CONCLUSIONES. VI. REFERENCIAS BIBLIOGRÁFICAS.

\section{INTRODUCCIÓN}

Para utilizar un medicamento las principales características que se deben considerar es la seguridad, eficacia y calidad. Para medicamentos tradicionales, de síntesis química, se ha considerado que demostrar bioequivalencia entre el medicamento original y un medicamento genérico es suficiente para que sean considerados intercambiables entre ellos. Las autoridades sanitarias deben considerar rigurosos estudios farmacocinéticos.

Los medicamentos biológicos son productos complejos, tiene grandes estructuras moleculares. Muchos son producidos utilizando diversas líneas celulares, pueden ser inestables 
y tiene diversas propiedades fisicoquímicas. Son susceptibles a los cambios ambientales y en el proceso de manufactura. En general es difícil determinar diferencias estructurales debidas a cambios en el proceso de manufactura entre un biosimilar o el producto de referencia ${ }^{1,2}$.

El presente trabajo aborda los conceptos fundamentales sobre la intercambiabilidad, medicamentos biotecnológicos y biosimilares, muestra el panorama internacional de cómo se ha abordado la intercambiabilidad de los medicamentos biosimilares.

\section{MEDICAMENTOS BIOLÓGICOS}

Los medicamentos biológicos, según la normativa vigente en Costa Rica, son productos obtenidos a partir de una variedad de fuentes naturales, de origen humano, animal o de microorganismos y, al igual que otros medicamentos, son utilizados para el alivio, tratamiento o prevención de enfermedades ${ }^{3}$.

Los medicamentos biológicos se distinguen de los medicamentos convencionales por ser derivados de organismos vivos y tienen una estructura molecular compleja. Estos productos requieren de consideraciones especiales debido a la naturaleza de los materiales de partida, al proceso de fabricación y a los métodos de análisis necesarios para la caracterización de los lotes del producto ${ }^{4}$. Es frecuente agrupar los productos biológicos en tres grandes grupos: vacunas, biológicos y biotecnológicos 5 .

El origen de los medicamentos biológicos difícil de esclarecer, para algunos la historia de los biológicos inicia en la época del desarrollo de la primera vacuna, cuando Edward Jenner, en 1796, inventó la vacuna contra la viruela ${ }^{6}$ y, posteriormente se sumaron los avances que logró en 1895, Louis Pasteur con el desarrollo de la vacuna contra la rabia. En 1902, inició el control de los medicamentos biológicos, ya que en ese momento el suero antidiftérico era el producto más importante, y se estaba incrementando el uso de otros sueros ${ }^{7}$. En 1934 se incluyeron en el panorama la "sangre y los productos derivados de la sangre" (hemoderivados) como resultado de su uso en las transfusiones de sangre.

Posteriormente, el desarrollo de la bacteriología y la obtención de los cultivos celulares, fueron claves para los siguientes avances en la fabricación de los productos biológicos, por ejemplo, las vacunas fraccionadas y purificadas. Se inició la comercialización de la vacuna contra la Bordetella pertussis acelular y la vacuna contra la influenza estacional.

\footnotetext{
${ }^{1}$ SEKHON, B.S., "Biosimilars: an overview". Biosimilars 2011; 1:1-11.

2 ADMINISTRACIÓN DE ALIMENTOS Y MEDICAMENTOS DE ESTADOS UNIDOS DE AMÉRICA. Draft guidance for industry - scientific considerations in demonstrating biosimilarity to a reference product. Center for Drug Evaluation and Research and Center for Biologics Evaluation and Research, US Food and Drug Administration, Rockville, MD, 2012. Disponible en: http://www.fda.gov/downloads/drugs/guidancecomplianceregulatoryinformation/guidances/ucm291128.pdf

${ }^{3}$ PODER EJECUTIVO DE COSTA RICA. Reglamento Técnico: RTCR 440: 2010. Reglamento de Inscripción y Control de Medicamentos Biológicos. Decreto Ejecutivo No. 37006-S. Publicado en el Diario Oficial La Gaceta Número 59, del 22 de marzo de 2012.

${ }^{4}$ WORLD HEALTH ORGANIZATION. Annex 2 Guidelines for national authorities on quality assurance for biological products. WHO Technical Report Series (822), 1992. 31-46.

${ }^{5}$ WORLD HEALTH ORGANIZATION. Biotherapeutics products. 2018. Disponible en: http://www.who.int/biologicals/biotherapeutics/biotherapeutic-products/en/

${ }^{6}$ CENTERS FOR DISEASE CONTROL AND PREVENTION. History of Smallpox. 2016. Disponible en: https://www.cdc.gov/smallpox/history/history.html

${ }^{7}$ VON SCHWERIN, A., STOFF, H. y WAHRIG, B. (2013). Biologics, a history of agents made from living organisms in the twentieth century. Londres: Pickering \& Chatto (Publishers) Limited, p. 3.
} 
En el caso de los productos biotecnológicos (o bioterapéuticos), algunos de ellos, inicialmente fueron obtenidos por métodos de producción convencional mediante su extracción de los tejidos y secreciones de origen humano o animal, como en el caso de la hormona de crecimiento humana y la insulina de origen porcino en los años 1921 a $1934{ }^{8}$. Posteriormente, con aplicación de la tecnología de ADN recombinante en los años setentas, se logró su producción en mayor escala, con una mayor pureza y menor riesgo de transmisión de agentes infecciosos 9 .

Otro logro importante de la biotecnología, ha sido el desarrollo de los anticuerpos monoclonales, una vez que se descubrió en 1975 la posibilidad de fusionar un linfocito B con una célula de mieloma, dando lugar a una célula llamada hibridoma con capacidad de producir inmunoglobulinas y proliferar indefinidamente, lo que permitió producir grandes cantidades de inmunoglobulinas idénticas y específicas para un determinado antígeno o blanco terapéutico ${ }^{10}$.

El siguiente nivel en el desarrollo de los productos biológicos ha sido la aplicación de la ingeniería genética y la biotecnología, para la obtención de productos para las terapias avanzadas, con miras, por ejemplo, a la corrección de la mutación de un gen. Estas estrategias terapéuticas innovadoras ofrecen nuevas oportunidades para algunas enfermedades raras relacionadas con la mutación de un gen que actualmente carecen de tratamientos eficaces.

En las últimas décadas, los avances científicos en biotecnología han incrementado el número de medicamentos biológicos de origen biotecnológico disponibles para tratar diferentes enfermedades, entre ellas diferentes tipos de cáncer, enfermedades inflamatorias crónicas como artritis reumatoide, artritis psoriásica, psoriasis, espondilitis anquilosante, y enfermedades inflamatorias del intestino ${ }^{11}$.

Estos medicamentos innovadores han tenido mucho éxito para el tratamiento de enfermedades y han aumentado los ingresos por ventas de las compañías farmacéuticas que las producen. El vencimiento de la protección por medio de patentes de estas moléculas complejas, ha producido un aumento en la industria biotecnológica para el desarrollo de productos denominados biosimilares.

Los medicamentos biosimilares son medicamentos biotecnológicos similares en términos de calidad, seguridad y eficacia, a un producto biotecnológico innovador o de referencia, previamente autorizado por una agencia reguladora ${ }^{12}$. Estos medicamentos no deben aportar estudios clínicos de seguridad y eficacia tan exhaustivos como el innovador para ser comercializados, sino que al demostrar su biosimilitud se asume que son comparables en términos de calidad, seguridad y eficacia que los innovadores.

\footnotetext{
${ }^{8}$ Ibídem.

9 EUROPEAN MEDICINES AGENCY. Advance Therapy medicinal products. 2018. Disponible en: http://www.ema.europa.eu/ema/index.jsp?curl=pages/regulation/general/general_content_000294.jsp\&mid=WC0b01 ac05800241e 0

${ }^{10}$ SOTO, J. Asma. "Los anticuerpos monoclonales que vienen”. Rev. Asma, 3(1), 2016, pp .69-81.

${ }^{11}$ TSURUTA, L., LOPES DOS SANTOS, M., y MORO, A., "Biosimilars advancements: moving on the future", en Biotechnol Prog, 31(5), 2015, pp. 1139-49.

${ }^{12}$ EMA/CHMP. EMA/CHMP/437/04 Rev 1. Guideline on Similar Biological Medicinal Products. 2015. Disponible en: https://www.ema.europa.eu/en/documents/scientific-guideline/guideline-similar-biological-medicinal-productsrev1_en.pdf
} 


\section{LA INTERCAMBIABILIDAD DE MEDICAMENTOS}

La mayoría de los países incorporados a la Organización Mundial del Comercio, otorgan a los fabricantes de medicamentos innovadores, protección de derechos exclusivos temporales para la explotación de estos productos, por medio del sistema de patentes y sistemas de protección de datos de prueba. Estos beneficios están concebidos como un estímulo de la innovación para los laboratorios fabricantes ${ }^{13}$. Cuando el periodo de protección vence, se empiezan a fabricar medicamentos genéricos o medicamentos similares.

Una vez caducadas las patentes y la protección de datos de prueba, las autoridades sanitarias de cada país permiten el registro de medicamentos similares o copias del original y aprueban su comercialización siempre que cumplan los requisitos que exige la normativa de cada país.

En el caso de los medicamentos de síntesis química la normativa nacional define como medicamento intercambiable como: "aquél producto que es terapéuticamente equivalente al producto de referencia y que puede ser intercambiado en la práctica clínica" ${ }^{\text {"14 }}$.

La mayoría de los genéricos que se venden en el mercado nacional no han demostrado la equivalencia terapéutica y, por lo tanto, no se pueden considerar intercambiables, sin embargo, en la práctica clínica, los medicamentos innovadores en muchas ocasiones son intercambiados por un medicamento registrado como similar o copia del original no intercambiable. Este intercambio se realiza en el proceso de prescripción y despacho de los medicamentos; muchas veces es resultado de confusión y omisión de la observancia de la regulación respectiva.

La Tabla 1 incluye las definiciones que utiliza la Agencia Europea de Medicamentos, de Intercambiabilidad de un medicamento, se plantea además la diferencia entre intercambiabilidad, intercambio y sustitución. El intercambio de los productos las efectúa el profesional en medicina y la sustitución del medicamento la realiza el profesional farmacéutico. Estas dos acciones son las que tienen impacto directo sobre el paciente al utilizar un medicamento genérico en lugar del innovador, y en la práctica clínica poco importa si el producto regulatoriamente es intercambiable.

Tabla 1. Definiciones de Intercambiabilidad, Intercambio y Sustitución del medicamento.

\begin{tabular}{|c|l|}
\hline Término & \multicolumn{1}{c|}{ Definición } \\
\hline $\begin{array}{c}\text { Intercambiabilidad } \\
\text { (Interchangeability) }\end{array}$ & $\begin{array}{l}\text { Se refiere a una designación regulatoria en el que un medicamento puede ser } \\
\text { cambiado por otro que se espera tenga el mismo efecto clínico en una condición } \\
\text { clínica dada, y que se debe dar para cada paciente por iniciativa o en acuerdo con } \\
\text { el médico prescriptor }\end{array}$ \\
\hline $\begin{array}{c}\text { Intercambio } \\
\text { (Switching) }\end{array}$ & $\begin{array}{l}\text { Es una acción del médico prescriptor donde intercambia un medicamento por otro } \\
\text { con la misma indicación terapéutica en pacientes que están sometidos a un } \\
\text { tratamiento. }\end{array}$ \\
\hline $\begin{array}{c}\text { Sustitución del medicamento } \\
\text { (Drug substitution) }\end{array}$ & $\begin{array}{l}\text { Es una acción del farmacéutico que dispensa un medicamento por otro equivalente } \\
\text { e intercambiables en la farmacia sin consultar al prescriptor. }\end{array}$ \\
\hline
\end{tabular}

Fuente: EMA/CHMP, 2015

\footnotetext{
${ }^{13}$ ORGANIZACIÓN PANAMERICANA DE LA SALUD. El Acceso a los Medicamentos de Alto Costo en las Américas: contexto, desafíos y perspectivas. Serie Técnica No. 1. Medicamentos esenciales, acceso, e innovación. 2009. Disponible en: https://www.paho.org/hq/index.php?option=com_content\&view=article\&id=2149:2008-elacceso-medicamentos-alto-costo-americas \&Itemid=1178\&lang=es

${ }^{14}$ PODER EJECUTIVO DE COSTA RICA. Reglamento para el registro sanitario de los medicamentos que requieren demostrar equivalencia terapéutica. Publicado en el Diario Oficial La Gaceta Número 149, del 04 de agosto de 2005.
} 
La demostración de la bioequivalencia de un medicamento genérico con un producto de referencia, suele ser generalmente adecuada y suficiente para inferir la equivalencia terapéutica e intercambiabilidad, entre el medicamento genérico y el producto de referencia ${ }^{15}$. Sin embargo, este enfoque establecido para los medicamentos genéricos no es adecuado para el desarrollo, la evaluación y la autorización de los medicamentos biosimilares, ya que los productos biotecnológicos consisten en proteínas más grandes, complejas, y difíciles de caracterizar.

La intercambiabilidad de los medicamentos biotecnológicos innovadores por los biosimilares ha generado una gran controversia relativa a las repercusiones que pueda tener para la salud de las personas. Y en este sentido, no hay ninguna normativa nacional o posición que se refiera claramente a la intercambiabilidad de los medicamentos innovadores por los biosimilares.

\section{III.1 Medicamento biotecnológico frente a un medicamento biosimilar}

Un medicamento biotecnológico, según el reglamento de Inscripción y Control de Medicamentos Biológicos, es proteína o ácido nucleico que se obtiene a partir de un organismo modificado con tecnología de ADN recombinante (ADNr) o tecnología de hibridoma o líneas celulares transformadas. Son medicamentos que han tenido mucho éxito como terapias más específicas y dirigidas a las células que causan diferentes tipos de cáncer, enfermedades inflamatorias crónicas como artritis reumatoide, artritis psoriásica, psoriasis, espondilitis anquilosante, y enfermedades inflamatorias del intestino, que a la fecha utilizaban tratamientos muy agresivos o para las cuales no existía tratamiento.

Con la finalización de la protección que otorgan las patentes de estos productos biotecnológicos innovadores, la industria biotecnológica dedicada a producir medicamentos biosimilares ha aumentado significativamente. Un medicamento biosimilar fue definido en la normativa como aquel medicamento biológico que ha demostrado mediante el ejercicio de biosimilitud que es similar en términos de calidad, seguridad y eficacia al medicamento biológico de referencia.

En términos generales, el medicamento biotecnológico para obtener la aprobación de comercialización por parte de la autoridad sanitaria debe demostrar, con su propia información la calidad, seguridad y eficacia del producto. El medicamento biosimilar debe demostrar que es similar en términos de calidad, seguridad y eficacia al medicamento biotecnológico innovador, por medio de un ejercicio de comparabilidad o biosimilitud, que describe las actividades, incluyendo el diseño y conducción de estudios (preclínicos, clínicos y de calidad) y evaluación de los datos. El objetivo de estos análisis es demostrar que un medicamento biológico tiene un perfil similar en términos de calidad, eficacia, seguridad e inmunogenicidad que el medicamento biológico de referencia.

Para la autorización de un medicamento biotecnológico innovador es necesario que el fabricante presente información de seguridad y eficacia, debe aportar estudios pre clínicos, estudios clínicos fase I, II, III y IV. Sin embargo, un medicamento biosimilar solo debe presentar información de seguridad y eficacia, estudios pre clínicos comparativos y estudios clínicos comparativos. Además de un ejercicio de biosimiltud relacionado a la calidad.

\footnotetext{
${ }^{15}$ ORGANIZACIÓN PANAMERICANA DE LA SALUD. Recomendaciones para la Evaluación de Productos Bioterapéuticos Similares (PBS). Red PARF Documento Técnico No. 7. Washington, D.C.: OPS. 2011. Disponible en: http://apps.who.int/medicinedocs/documents/s19941es/s19941es.pdf
} 


\section{III.2 Intercambiabilidad de los medicamentos biotecnológicos}

La presencia en el mercado de medicamentos biotecnológicos innovadores y biosimilares, ha generado el debate en la comunidad científica sobre la intercambiabilidad de estos medicamentos entre sí. Como lo indica César Hernández-García: en este debate se mezclan a menudo, argumentos e incertidumbres científicas, posiciones regulatorias que necesariamente evolucionan a la luz de los conocimientos, elementos presupuestarios, puntos de vista interesados y desinteresados, en definitiva, mitos y realidades ${ }^{16}$.

La eficacia, seguridad y el riesgo de inmunogenicidad de intercambiar un medicamento biológico por un biosimilar o por otro innovador ha generado amplias discusiones, McKinnon et al, en $2018^{17}$, realizó una revisión sistemática de los resultados de estos intercambios 63 publicaciones. El estudio encontró que existen vacíos de evidencia sobre la seguridad del cambio entre los productos biológicos y sus biosimilares. Se necesitan ensayos clínicos y estudios de farmacovigilancia suficientemente potentes y analizados estadísticamente, con seguimientos a largo plazo y múltiples variaciones, para respaldar la toma de decisiones sobre la intercambiabilidad el cambio de biosimilares.

Una de las ventajas que se plantea de los biosimilares, es el ahorro en los costos de estos productos, sin embargo, existe la percepción que los biosimilares no tienen la misma calidad que el medicamento de referencia y que son medicamentos de una calidad menor al medicamento innovador. Como consecuencia de esta disminución de costos, se cree que puede poner en riesgo la salud de los pacientes. Sin embargo, para el registro de un medicamento biosimilar se debe demostrar comparativamente que su calidad, seguridad y eficacia es similar que la del medicamento biotecnológico innovador.

Es posible considerar que los medicamentos biosimilares son "similares" al medicamento biotecnológico de referencia, pero no "idénticos", sin embargo, los medicamentos biológicos son moléculas complejas que se producen en los sistemas vivos (líneas celulares), por lo que la variabilidad del sistema biológico y el proceso de fabricación confiere una variabilidad intrínseca (o inherente) en todos los medicamentos biotecnológicos, lo cual aplica también al medicamento de referencia, y ni siquiera un nuevo lote del medicamento biológico de referencia es, por lo tanto, idéntico al lote anterior o al siguiente. Por esta razón los requisitos reglamentarios para los medicamentos biotecnológicos (no solo los biosimilares) son muy estrictos en términos de control de parámetros críticos de calidad y validación del proceso de fabricación que asegure la reproducibilidad y cumplimiento de dichos parámetros lote a lote.

Como se mencionó anteriormente, la intercambiabilidad corresponde a una designación regulatoria en el que un medicamento puede ser cambiado por otro que se espera tenga el mismo efecto clínico en una condición clínica dada.

\section{III.3 El caso de la Eritropoyetina}

El sistema público de salud costarricense, provee a la población de medicamentos a través de la Caja Costarricense de Seguro Social (CCSS). La institución utiliza regularmente el

\footnotetext{
${ }^{16}$ FERNÁNDEZ-GARCÍA, C., "Mitos y realidades sobre los medicamentos biosimilares, en Reumatología Clínica, 10 (6), 2014, pp. 351-352.

${ }^{17}$ MCKINNON, R.A., COOK, M., LIAUW, W., MARABANI, M. MARSCHNER, I.C., PACKER, N. H., PRINS, J. B. "Biosimilarity and Interchangeability: Principles and Evidence: A Systematic Review", en BioDrugs 32. 2018, pp. $27-52$.
} 
medicamento denominado Eritropoyetina, este producto es un medicamento biológico indicado en el tratamiento de la anemia sintomática asociada a la insuficiencia renal crónica en pacientes adultos y pediátricos.

Desde el mes de enero de 2016 se incrementó las notificaciones de sospechas de reacciones adversas. En el Centro Nacional de Farmacovigilancia existe un total de 68 reportes de sospechas de reacciones adversas durante el período de junio de 2005 a mayo de 2016. De estas 68 notificaciones, 54 notificaciones corresponden al período de enero a mayo de 2016. Durante el primer semestre de 2016, la eritropoyetina fue reportada 58 veces $^{18}$.

Este aumento de notificaciones coincide con el inicio de la utilización, por parte de la CCSS del medicamento eritropoyetina alfa (Epocim®) 2000 UI/ML del laboratorio Centro de Inmunología Molecular de Cuba (CIMAB). Hasta el año 2016, la CCSS utilizaba la eritropoyetina beta, Fabricado por el Laboratorio Roche, bajo el nombre de marca Recormon. Desde que se cambió el medicamento los pacientes experimentan reacciones adversas como dolor en la aplicación y eritema a tal punto que los pacientes se rehúsan a aplicarse nuevamente el medicamento. En diferentes hospitales del país (Hospital San Juan de Dios, Hospital Calderón Guardia y Hospital México), se reportaron fallas terapéuticas.

La comercialización del medicamento Epocim® (Eritropoyetina alfa) se realizó antes del 2012, fecha en la que se promulgó el Reglamento de Inscripción y Control de Medicamentos Biológicos. Para la autorización de este producto no se solicitó la comparación con un producto de referencia original. Para la autorización de este producto no se solicitó la comparación con un producto de referencia original pues no era requerimiento de registro; sin embargo, durante la renovación del registro sanitario este producto debía cumplir con la normativa vigente para medicamentos biológicos, y no logró demostrar la calidad, seguridad y eficacia comparativa con el medicamento innovador puesto que no se le renovó su autorización en ante el Ministerio de Salud.

A pesar de la falta de evidencia científica y de parámetros de seguridad y eficacia, la CCSS utilizaba para sus pacientes con insuficiencia renal crónica, de manera equivalente la eritropoyetina alfa; con la Eritropoyetina beta. Sin embargo, el caso nos permite reflexionar sobre las consecuencias que puede haber para la salud si se sustituye un medicamento por otro que no ha demostrado una calidad, seguridad y eficacia comparativa con el medicamento innovador y la importancia de la farmacovigilancia en los sistemas de salud.

\section{REGULACIÓN DE LA INTERCAMBIABILIDAD DE LOS MEDICAMENTOS BIOTECNOLÓGICOS BIOSIMILARES EN LA UNIÓN EUROPEA Y AMÉRICA}

La Organización Panamericana de la Salud, ha reconocido la necesidad que las autoridades nacionales definan diferentes aspectos importantes asociados con el uso de los medicamentos biotecnológicos biosimilares, y uno de esos aspectos es la intercambiabilidad y sustitución del medicamento biotecnológico innovador y el biosimilar ${ }^{19}$.

\footnotetext{
${ }^{18}$ MINISTERIO DE SALUD DE COSTA RICA. Dirección De Regulación de Productos de Interés Sanitario Centro Nacional de Farmacovigilancia. Informe I Semestre de 2016. 2016. Disponible en https://www.ministeriodesalud.go.cr/index.php/informes-y-estadisticas/3115-informe-primer-semestre-de-2016sospechas-de-reaccion-adversa-a-medicamento/file

19 ORGANIZACIÓN PANAMERICANA DE LA SALUD. Recomendaciones para la Evaluación de Productos Bioterapéuticos Similares (PBS). Red PARF, Documento Técnico No. 7. Washington, D.C.: OPS. 2011.
} 
En la Unión Europea, cuando la Agencia Europea de Medicamentos realiza la evaluación de la información científica de un biosimilar es para determinar si dicho producto es similar en términos de calidad, seguridad y eficacia con el medicamento biotecnológico de referencia, en dicha evaluación no se incluyen recomendaciones de si el medicamento biosimilar es intercambiable o no con el producto de referencia. La decisión de si un producto puede ser intercambiable o no por otro, es una decisión que debe ser tomada a nivel nacional en cada uno de los estados miembros ${ }^{20}$.

En Estados Unidos, con la Ley de Competitividad de precios e Innovación de Biológicos (Biologics Price Competition and Innovation (BPCI) Act) en el 2009 se introdujo el concepto de intercambiabilidad de un biosimilar por el medicamento innovador; sin embargo, para que un medicamento biosimilar sea denominado intercambiable por la FDA dicho medicamento debe demostrar un alto grado de comparabilidad con el medicamento innovador y presentar un estudio clínico donde demuestre la intercambiabilidad. A la fecha la FDA no ha aprobado ningún medicamento biosimilar intercambiable.

De acuerdo a la FDA son productos intercambiables tanto el biosimilar como el producto de referencia aprobado por la FDA, y se puede esperar que produzca el mismo resultado clínico del producto de referencia en cualquier paciente dado. Un producto intercambiable puede sustituir al producto de referencia sin la intervención del proveedor de salud quien prescribió el producto de referencia. Además, para productos biológicos que deban ser administrados más de una vez a un paciente, el riesgo en términos de seguridad y eficacia de alternar o intercambiar entre el producto biológico y el producto de referencia no será mayor que el riesgo de usar el producto de referencia sin la alternación o intercambio ${ }^{21}$.

Lo cual requiere que el producto a parte de demostrar la biosimilitud con el producto de referencia debe presentar un estudio clínico de intercambiabilidad; sin embargo, a la fecha ninguno de los biosimilares aprobados por la FDA ha demostrado intercambiabilidad con el producto de referencia.

La autoridad reguladora de Canadá, Health Canada, de manera similar a la Agencia Europea, solo evalúa si un producto se considera biosimilar al medicamento biológico de referencia, no declara si dos productos son intercambiables o no, esta responsabilidad recae sobre cada una de las provincias de acuerdo a sus propias reglas y regulaciones ${ }^{22}$.

En Latinoamérica, en México, Brasil, Chile, Panamá y Costa Rica, la situación es similar para definir si un producto medicamento biotecnológico innovador es intercambiable por el biosimilar no ha sido definido o reglamentado.

En México la Norma Oficial Mexicana NOM-177-SSA1-2013 establece las pruebas y procedimientos para demostrar que un medicamento genérico es intercambiable, así como los requisitos para demostrar la biocomparabilidad de los medicamentos biológicos, por lo que al igual que las autoridades reguladoras de EMA, Health Canada, la Comisión Federal para la Protección contra Riesgos Sanitarios (COFEPRIS) en México evalúa y aprueba un

\footnotetext{
${ }^{20}$ DALLER, J., "Biosimilars: A consideration of the regulations in the United States and European Union", en Regulatory Toxicology and Pharmacology, 76, 2015, pp. 199-208.

${ }^{21}$ FOOD AND DRUG ADMINISTRATION. Considerations in demostrating Interchangeability With a Reference Product. 2017. Disponible en:

https://www.fda.gov/downloads/Drugs/GuidanceComplianceRegulatoryInformation/Guidances/UCM537135.pdf

22 HEALTH CANADA. Fact Sheet: Biosimilars. 2017. Disponible en: https://www.canada.ca/en/healthcanada/services/drugs-health-products/biologics-radiopharmaceuticals-genetic-therapies/applicationssubmissions/guidance-documents/fact-sheet-biosimilars.html
} 
medicamento biocomparable (biosimilar) a un medicamento biotecnológico innovador pero no hay regulación para determinar si son intercambiables ${ }^{23}$.

En Brasil, la regulación para la aprobación de medicamentos biosimilares por parte de Agencia Nacional de Vigilancia Sanitaria (ANVISA), solo asegura la biosimilitud entre el biosimilar y el producto biológico de referencia, pero no regula la intercambiabilidad de estos productos. Ante el debate de la intercambiabilidad en el 2017 ANVISA emitió una nota pública aclaratoria donde se explica que, en el entendido de la Agencia, la decisión de intercambiar el producto de biológico innovador por el biosimilar recae sobre el médico ${ }^{24}$.

En Chile, la Norma Técnica No. 170 del Instituto de Salud Pública establece claramente que la autorización sanitaria de un producto biosimilar, posibilita intercambiabilidad o sustitución entre productos, siempre que se realice mediante un proceso escalonado, procedimentado y bajo estrecha supervisión médica. La intercambiabilidad se realizará, por decisión del médico quien evaluará los riesgos y beneficios e informará al paciente sobre estos últimos. Los pacientes deberán estar en conocimiento de la sustitución de sus medicamentos biotecnológicos ${ }^{25}$.

En Panamá la Ley 1 del 10 de enero de 2001 indica que la Autoridad de Salud reglamentará y certifica lo relacionado con los medicamentos intercambiables, para lo cual elaborará y publicará un Listado de Medicamentos Intercambiables ${ }^{26}$, pero esto al igual que en la mayoría de las autoridades aplica para medicamentos de síntesis química. De acuerdo a la Dirección Nacional de Farmacia y Drogas la intercambiabilidad no aplica para los productos biológicos o no está regulada; sin embargo, existe una regulación sobre la sustitución de los productos, y no se permite la sustitución de los medicamentos biológicos ${ }^{27}$, pero actualmente en la lista de inicial de medicamentos no sustituibles que se encuentra publicada únicamente incluye al medicamento biotecnológico con principio activo basilixumab ${ }^{28}$.

En Costa Rica, de manera similar a la mayoría de las autoridades sanitarias que se han analizado en esta sección, el Ministerio de Salud cuando autoriza un medicamento biosimilar es porque dicho medicamento ha demostrado ser comparable al medicamento biotecnológico de referencia en términos de calidad, seguridad y eficacia de acuerdo a la normativa vigente, la cual no se refiere a la intercambiabilidad de dichos medicamentos.

\footnotetext{
${ }^{23}$ SECRETARÍA DE SALUD DE MÉXICO. Norma Oficial Mexicana NOM-177-SSAI-2013. Publicada en el Diario Oficial de la Federación el 20 de septiembre de 2013.

${ }^{24}$ ASCOM/ANVISA. Biossimilares são intercambiáveis? 2018. Disponible en: http://portal.anvisa.gov.br/noticias//asset_publisher/FXrpx9qY7FbU/content/biossimilares-sao-intercambiaveis-/219201/pop_up?inheritRedirect=false

${ }^{25}$ MINISTERIO DE SALUD REPÚBLICA DE CHILE. Norma Técnica No. 170 Sobre el Registro Sanitario de Productos Biotecnológicos Derivados de Técnicas ADN Recombinantes. 2014. Disponible en: http://www.ispch.cl/sites/default/files/Norma\%20Biotecnologicos.pdf

${ }^{26}$ ASAMBLEA LEGISLATIVA DE PANAMÁ. Ley 1 de 10 de enero de 2001 Sobre Medicamentos y otros Productos para la Salud Humana. Gaceta Oficial (No. 24218). Disponible en: http://www.minsa.gob.pa/sites/default/files/publicacion-general/ley-1-de-2001-sobre-medicamentos.pdf

${ }^{27}$ MINISTERIO DE SALUD REPÚBLICA DE PANAMÁ. Decreto Ejecutivo No. 105 de 2 de mayo de 2017 Que regula la prescripción y dispensación de medicamentos nos sustituibles. Gaceta Oficial (No. 28279 B). Disponible en: https://www.gacetaoficial.gob.pa/pdfTemp/28279_B/61123.pdf

${ }^{28}$ DIRECCIÓN NACIONAL DE FARMACIA Y DROGAS. Resolución No. 355 de 15 de junio de 2017 Lista Inicial de Medicamentos No Sustituibles al 15 de Junio de 2017. Gaceta Oficial (No. 28303-A). Disponible en: https://www.gacetaoficial.gob.pa/pdfTemp/28303_A/GacetaNo_28303a_20170619.pdf
} 


\section{CONCLUSIONES}

En la práctica médica el intercambio de un medicamento innovador por otro medicamento conteniendo en mismo principio activo se da por prácticas como el intercambio o sustitución del medicamento y no necesariamente porque el medicamento sea intercambiable, y esto sucede tanto para medicamentos de síntesis química como medicamentos biológicos.

El caso de la eritropoyetina demuestra la consecuencia que puede tener, para los pacientes, realizar un intercambio de medicamentos sin las debidas precauciones, y la importancia de la farmacovigilancia como herramienta en la salud pública.

La intercambiabilidad de los medicamentos biotecnológicos innovadores por los biosimilares continúa generando gran controversia relativa a las repercusiones que pueda tener para la salud de las personas y necesidad de los sistemas de salud por obtener medicamentos de calidad, seguros y eficaces a un menor costo.

\section{REFERENCIAS BIBLIOGRÁFICAS}

- Administación de alimentos y medicAmentos de Estados Unidos de América (2012). "Draft guidance for industry - scientific considerations in demonstrating biosimilarity to a reference product". Center for Drug Evaluation and Research and Center for Biologics Evaluation and Research, US Food and Drug Administration, Rockville, MD. Disponible en:

http://www.fda.gov/downloads/drugs/guidancecomplianceregulatoryinformation/guidances/ ucm291128.pdf

- Asamblea Legislativa de Panamá. Ley 1 de 10 de enero de 2001 Sobre Medicamentos y otros Productos para la Salud Humana. Gaceta Oficial (No. 24218). Disponible en: http://www.minsa.gob.pa/sites/default/files/publicacion-general/ley-1-de-2001-sobremedicamentos.pdf

- Ascom/Anvisa (2018). Biossimilares são intercambiáveis? Disponible en: http://portal.anvisa.gov.br/noticias/-/asset_publisher/FXrpx9qY7FbU/content/biossimilaressao-intercambiaveis-/219201/pop_up?inheritRedirect=false

- CENTERS FOR DiSEASE CONTROL AND PREVENTION (2016). History of Smallpox. Disponible en: https://www.cdc.gov/smallpox/history/history.html

- DALleR, J. (2015). "Biosimilars: A consideration of the regulations in the United States and European Union”. Regulatory Toxicology and Pharmacology, 76, 2015. 199-208.

- Dirección NACIONAl De FARMACIA Y DROGas. Resolución No. 355 de 15 de junio de 2017. Lista Inicial de Medicamentos No Sustituibles al 15 de Junio de 2017. Gaceta Oficial (No. 28303-A). Disponible en: https://www.gacetaoficial.gob.pa/pdfTemp/28303_A/GacetaNo_28303a_20170619.pdf

- EMA/CHMP/437/04 (2015). Rev 1. Guideline on Similar Biological Medicinal Products. Disponible en: https://www.ema.europa.eu/en/documents/scientific-guideline/guidelinesimilar-biological-medicinal-products-rev1_en.pdf

- European Medicines Agency (2018). Advance Therapy medicinal products. Disponible en: http://www.ema.europa.eu/ema/index.jsp?curl=pages/regulation/general/general_content_0 00294.jsp\&mid=WC0b01ac05800241e0 
- Fernández GARCÍA, C. (2014). "Mitos y realidades sobre los medicametnos biosimilares". Reumatología Clínica, 10 (6), 2014, pp. 351-352.

- FOOD AND DRUG ADMINISTRATION (2017). Considerations in demostrating Interchangeability With a Reference Product. Disponible en: https://www.fda.gov/downloads/Drugs/GuidanceComplianceRegulatoryInformation/Guidan ces/UCM537135.pdf

- Health CANAdA (2017). Fact Sheet: Biosimilars. Disponible en: https://www.canada.ca/en/health-canada/services/drugs-health-products/biologicsradiopharmaceuticals-genetic-therapies/applications-submissions/guidance-documents/factsheet-biosimilars.html

- McKinnon, R.A., Cook, M., Liauw, W., Marabani, M., Marschner, I.C., Packer, N.H., PRINS, J.P. (2018). "Biosimilarity and Interchangeability: Principles and Evidence: A Systematic Review”. BioDrugs 32. 2018, pp. 27-52.

- Ministerio de Salud de Costa Rica (2016). Dirección de Regulación de Productos de Interés Sanitario Centro Nacional de Farmacovigilancia. Informe I Semestre de 2016. Disponible en https://www.ministeriodesalud.go.cr/index.php/informes-y-estadisticas/3115informe-primer-semestre-de-2016-sospechas-de-reaccion-adversa-a-medicamento/file

- Ministerio de Salud República de Chile (2014). Norma Técnica No. 170 Sobre el Registro Sanitario de Productos Biotecnológicos Derivados de Técnicas ADN Recombinantes. Disponible en:

http://www.ispch.cl/sites/default/files/Norma\%20Biotecnologicos.pdf

- Ministerio de Salud República de Panamá (2017). Decreto Ejecutivo No. 105 de 2 de Mayo de 2017 que regula la prescripción y dispensación de medicamentos nos sustituibles. Gaceta Oficial (No. 28279 B). Disponible en: https://www.gacetaoficial.gob.pa/pdfTemp/28279_B/61123.pdf

- ORganización PANAmericana DE la SAlud (2009). "El Acceso a los Medicamentos de Alto Costo en las Américas: contexto, desafíos y perspectivas". Serie Técnica No. 1. Medicamentos esenciales, acceso, e innovación. Disponible en:

https://www.paho.org/hq/index.php?option=com_content\&view=article\&id=2149:2008-elacceso-medicamentos-alto-costo-americas \&Itemid=1178\&lang=es

- ORganización PANAmericana DE LA SAlud (2011). "Recomendaciones para la Evaluación de Productos Bioterapéuticos Similares (PBS)". Red PARF Documento Técnico No. 7. Washington, D.C.: OPS. 2011. Disponible en: http://apps.who.int/medicinedocs/documents/s19941es/s19941es.pdf

- Poder EjeCutivo de Costa RiCa (2005). Reglamento para el registro sanitario de los medicamentos que requieren demostrar equivalencia terapéutica. Publicado en el Diario Oficial La Gaceta Número 149, del 04 de agosto de 2005.

- Poder Ejecutivo de Costa Rica (2012). Reglamento Técnico: RTCR 440: 2010. Reglamento de Inscripción y Control de Medicamentos Biológicos. Decreto Ejecutivo No. 37006-S. Publicado en el Diario Oficial La Gaceta Número 59, del 22 de marzo de 2012.

- Secretaría de SAlud de MéXico (2013). Norma Oficial Mexicana NOM-177-SSAI2013. Publicada en el Diario Oficial de la Federación el 20 de septiembre de 2013.

- SEKHON, B.S. (2011). "Biosimilars: an overview". Biosimilars 2011; 1:1-11.

- Soto, J. (2016). “Asma. Los anticuerpos monoclonales que vienen”. Rev Asma, 3(1), 2016, pp. $69-81$.

- Tsuruta, L., Lopes Dos Santos, M. y Moro, A. (2015). "Biosimilars advancements: moving on the future". Biotechnol Prog, 31(5), 2015, pp. 1139-49. 
- Von Schwerin, A., StOFF, H. Y WAHRIG, B. (2013). Biologics, a history of agents made from living organisms in the twentieth century. Londres: Pickering \& Chatto (Publishers) Limited, pp. 3.

- World Health Organization (1992). Annex 2 Guidelines for national authorities on quality assurance for biological products. WHO Technical Report Series (822), 1992. 3146.

- World Health ORganization (2018). Biotherapeutics products. Disponible en: http://www.who.int/biologicals/biotherapeutics/biotherapeutic-products/en/ 